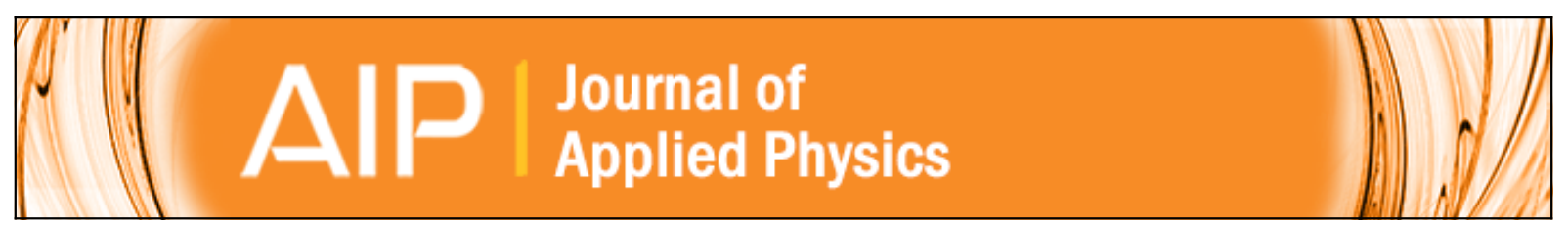

\title{
Thermoelectric properties of indium doped PbTe1-ySey alloys
}

Ashoka Bali, Heng Wang, G. Jeffrey Snyder, and Ramesh Chandra Mallik

Citation: Journal of Applied Physics 116, 033707 (2014); doi: 10.1063/1.4890320

View online: http://dx.doi.org/10.1063/1.4890320

View Table of Contents: http://scitation.aip.org/content/aip/journal/jap/116/3?ver=pdfcov

Published by the AIP Publishing

\section{Articles you may be interested in}

Importance of ligands in the electronic properties of FeTe0.6Se0.4

J. Appl. Phys. 114, 163906 (2013); 10.1063/1.4827192

Correlation between microstructure and thermoelectric properties of AgPb18SbTe20 (LAST-18)

AIP Conf. Proc. 1449, 171 (2012); 10.1063/1.4731524

High-output-power densities from molecular beam epitaxy grown n- and p-type PbTeSe-based thermoelectrics via improved contact metallization

J. Appl. Phys. 111, 104501 (2012); 10.1063/1.4712425

The QuantumFunctional Properties of Pr1xyLaxPbyTe

AIP Conf. Proc. 850, 1291 (2006); 10.1063/1.2355179

Macroscopic thermoelectric inhomogeneities in ( $\mathrm{Ag} \mathrm{Sb} \mathrm{Te} 2) \times(\mathrm{Pb}$ Te $) 1 \times$

Appl. Phys. Lett. 87, 171903 (2005); 10.1063/1.2056590

A|P| $\left.\right|_{\text {Letters }} ^{\text {Applied Physics }}$

is pleased to announce Reuben Collins as its new Editor-in-Chief

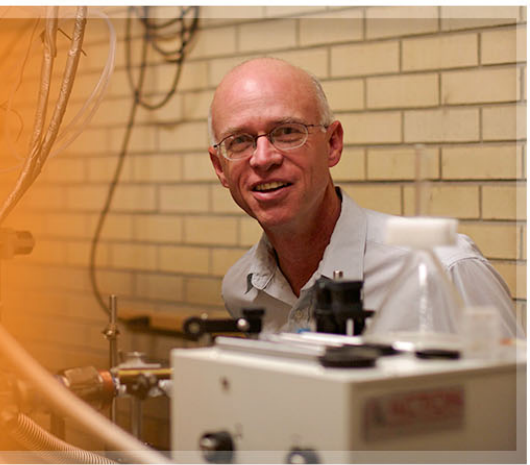




\title{
Thermoelectric properties of indium doped $\mathrm{PbTe}_{1-\mathrm{y}} \mathrm{Se}_{\mathrm{y}}$ alloys
}

\author{
Ashoka Bali, ${ }^{1}$ Heng Wang, ${ }^{2}$ G. Jeffrey Snyder, ${ }^{2}$ and Ramesh Chandra Mallik ${ }^{1, a)}$ \\ ${ }^{1}$ Thermoelectric Materials and Devices Laboratory, Department of Physics, Indian Institute of Science, \\ Bangalore 560012, Karnataka, India \\ ${ }^{2}$ Department of Materials Science, California Institute of Technology, Pasadena, California 91125, USA
}

(Received 31 May 2014; accepted 3 July 2014; published online 18 July 2014)

\begin{abstract}
Lead telluride and its alloys are well known for their thermoelectric applications. Here, a systematic study of $\mathrm{PbTe}_{1-\mathrm{y}} \mathrm{Se}_{\mathrm{y}}$ alloys doped with indium has been done. The powder X-Ray diffraction combined with Rietveld analysis confirmed the polycrystalline single phase nature of the samples, while microstructural analysis with scanning electron microscope results showed densification of samples and presence of micrometer sized particles. The temperature dependent transport properties showed that in these alloys, indium neither pinned the Fermi level as it does in PbTe, nor acted as a resonant dopant as in SnTe. At high temperatures, bipolar effect was observed which restricted the $z \mathrm{~T}$ to 0.66 at $800 \mathrm{~K}$ for the sample with $30 \%$ Se content. C 2014 AIP Publishing LLC. [http://dx.doi.org/10.1063/1.4890320]
\end{abstract}

\section{INTRODUCTION}

The need for alternative sources of energy has led to an interest in thermoelectric materials, where direct conversion of heat to electricity, and vice-versa is possible. The efficiency of this conversion depends on a dimensionless figure of merit $(z \mathrm{~T})=\mathrm{S}^{2} \mathrm{~T} / \rho \kappa_{\text {total }}$, where $\mathrm{S}$ is the Seebeck coefficient, $\rho$ is the electrical resistivity, $\mathrm{T}$ the temperature and $\kappa_{\text {total }}\left(=\kappa_{\mathrm{e}}+\kappa_{1}\right)$, is the total thermal conductivity of the material, with $\kappa_{\mathrm{e}}$ as the electronic and $\kappa_{1}$ the lattice contribution. Lead telluride (PbTe) and its compounds have been used as mid temperature $(300-850 \mathrm{~K})$ thermoelectric materials with a $z \mathrm{~T}$ of $\sim 0.8^{1}$ The material parameter $(\beta)$, defined by

$$
\beta=\left(\frac{k_{B}}{e}\right)^{2} \frac{(2 e \mu)}{\kappa_{l}}\left(\frac{2 \pi m^{*} k_{B} T}{h^{2}}\right)^{3 / 2} T,
$$

where $\mu$ is the mobility, $\mathrm{k}_{\mathrm{B}}$ the Boltzmann constant, e the electronic charge, $\kappa_{1}$ the lattice thermal conductivity, $\mathrm{m} *$ the density of states effective mass given by $\mathrm{N}_{\mathrm{v}}{ }^{2 / 3}\left(\mathrm{~m}_{\perp}^{* 2} \mathrm{~m}^{*} \|\right)^{1 / 3}$, $\mathrm{N}_{\mathrm{v}}$ is the band degeneracy and $\mathrm{m}^{*}{ }_{\perp}$ and $\mathrm{m}^{*} \|$ are effective masses in the transverse and the longitudinal directions, respectively, is related to $z \mathrm{~T}$. At any temperature, depending on the scattering mechanism, all the properties except $\kappa_{1}$ are electronic properties, which depend on the carrier concentration. $\beta$ can be increased by reducing $\kappa_{1}$ by alloying it with an element from the same group at either site of the PbTe crystal- $\mathrm{Pb}$ or Te. Secondly alloying also results in a change of band structure like tuning of band gap (e.g. in $\mathrm{PbSn}_{\mathrm{x}} \mathrm{Te}_{1-\mathrm{x}}$ alloys $^{2}$ ), convergence of bands, ${ }^{3}$ etc. PbTe-PbSe alloys, where Te is substituted by isovalent Se, have been studied several times in the past. ${ }^{4-8} \mathrm{~A}$ band degeneracy of 12 was reached by convergence of many valleys in valence band at high temperatures in p-type $\mathrm{PbTe}_{1-\mathrm{y}} \mathrm{Se}_{\mathrm{y}}$ material resulting in high $z \mathrm{~T}$ of 1.8 at $850 \mathrm{~K}^{3}$ In another report, $z \mathrm{~T}=1.35$ at $675 \mathrm{~K}$ (Ref. 6) was obtained by adding 0.7 at. \% Na. On the

\footnotetext{
a) Author to whom correspondence should be addressed. Electronic mail: rcmallik@physics.iisc.ernet.in
}

other hand, n-type alloys have shown lower $z \mathrm{~T},{ }^{9}$ primarily due to the absence of any such band modification. Moreover, in both the cases, the high value of $z \mathrm{~T}$ is obtained only at high temperatures, whereas for practical applications, high $z \mathrm{~T}$ in the entire operation range is desirable. Hence, for achieving better n-type $\mathrm{PbTe}_{1-\mathrm{y}} \mathrm{Se}_{\mathrm{y}}$ alloys, additional doping is required to be done in order to control the above mentioned electronic properties. Even though there are several well established n-type dopants in PbTe, ${ }^{10,11}$ indium (In) has been found to lead to a stabilization of carrier concentration up to 2 at. \% In content, which happens due to Fermi level pinning. ${ }^{12,13}$ This could also be useful for $\mathrm{PbTe}_{1-\mathrm{y}} \mathrm{Se}_{\mathrm{y}}$ alloys for achieving homogeneity of transport properties over a larger temperature range. Second, even though other group III elements like $\mathrm{Tl}$ have been found to form resonant level in PbTe, ${ }^{14}$ In has not been found to do so in either PbTe or $\mathrm{PbSe}$. However, since alloying leads to a change of band gap and also the band structure, there is a possibility of doing so, which could lead to increase in Seebeck coefficient. Further, the effect of substituting $\mathrm{In}$ at $\mathrm{Pb}$ site and $\mathrm{Se}$ at Te site simultaneously on the band parameters and their consequent effect on thermoelectric properties have not been studied so far.

In this paper, a systematic study of PbTe-PbSe system with varying Se and fixed In content has been done. PbTe and $\mathrm{PbSe}$ have been known to form solid solution over a large composition range. ${ }^{5}$ Here, a maximum of $30 \%$ Se was chosen for the PbTe-PbSe alloy system. For indium, its phase diagram shows that InTe is soluble in PbTe upto 7 mol. \% (Ref. 15) with single phase structure. Here, a low concentration of 0.1 at. $\%$ In was chosen, which falls well within this region. No supplementary doping with halogens was done so as to study the control of carrier concentration only by indium and the off-stoichiometry from PbTe. It was found that the carrier concentration of the samples varied in a narrow range since Se did not contribute to any extra electrons to the system and a maximum $z \mathrm{~T}$ of 0.66 was reached at $800 \mathrm{~K}$. 


\section{EXPERIMENTAL DETAILS}

$\mathrm{Pb}_{0.999} \mathrm{In}_{0.001} \mathrm{Te}_{1-\mathrm{y}} \mathrm{Se}_{\mathrm{y}}(\mathrm{y}=0.01,0.05,0.10,0.20,0.25$, and 0.30 , labeled as VA1, VA2, VA3, VA4, VA5, and VA6, respectively) was prepared from high purity $\mathrm{Pb}(99.9 \%$, $\mathrm{Te}(99.999 \%), \operatorname{In}(99.99 \%)$, and $\operatorname{Se}(99.999 \%)$. The elements were mixed in appropriate stoichiometric ratios and sealed under $1.3 \times 10^{-2} \mathrm{~Pa}$ vacuum in carbon coated quartz tubes. The ampoules were then slowly heated upto $1273 \mathrm{~K}$ in $10 \mathrm{~h}$ and soaked at that temperature for $6 \mathrm{~h}$, which was followed by quenching in water. The samples were then annealed at $973 \mathrm{~K}$ for $72 \mathrm{~h}$, after which water quenching was done again. The ingots thus prepared were subjected to hand grinding with mortar and pestle and compacted into cylindrical pellets by hot pressing under vacuum $\sim 7 \times 10^{-5}$ torr at $913 \mathrm{~K}$ with $30 \mathrm{MPa}$ pressure for $30 \mathrm{~min}$. Two identical pellets were prepared for each sample - one was used for transport properties measurement, while the other was used for microstructural characterisation. The pellets used for transport property measurement were $14 \mathrm{~mm}$ diameter and thinned down to $1 \mathrm{~mm}$ thickness. All the pellets had more than $95 \%$ of the theoretical density $\left(8.24 \mathrm{~g} / \mathrm{cm}^{3}\right)$.

Phase identification was done by Bruker D8 Advance with $\mathrm{Cu} \mathrm{K}_{\alpha}$ radiation with $\mathrm{Si}$ as an internal standard and scan speed of $2 \%$ min. Backscattered electron images (BSEs) were taken with Quanta 200 environmental scanning electron microscope (ESEM). Compositional analysis was carried out on JEOL JXA-8530F Electron Probe Micro Analyzer (EPMA) with Wavelength Dispersive Spectroscopy (WDS) detectors. Temperature dependent Seebeck coefficient was measured using the apparatus described in Ref. 16. The inplane Hall coefficient and electrical resistivity were measured using the Van der Pauw method under $\pm 2 \mathrm{~T}$ magnetic field. Thermal diffusivity (D) was measured by laser flash method (Netzsch LFA 457) on the same pellets. Prior to carrying out the measurement, the pellets were coated with graphite spray for better heat conductance. The specific heat $\left(\mathrm{C}_{\mathrm{p}}\right)$ was calculated using the empirical formula $\mathrm{C}_{\mathrm{p}} / \mathrm{k}_{\mathrm{B}}=3.07+4.7 \times 10^{-4}(\mathrm{~T} / \mathrm{K}-300)$, which is based on experimental results and has been used earlier for lead chalcogenides. ${ }^{3,7,10,11,17}$ The thermal conductivity was calculated from $\kappa=\mathrm{C}_{\mathrm{p}} \times \mathrm{d} \times \mathrm{D}$, where $\mathrm{d}$ is the density. For each of the transport property measurement, the uncertainty was $5 \%$.

\section{RESULTS AND DISCUSSION}

\section{A. Material characterisation}

Figure 1(a) shows the X-ray diffraction pattern of all the samples. Sharp peaks indicative of polycrystalline nature of the samples were observed. The peaks were indexed according to ICSD \#182660 for PbTe since both the binary end compounds- $\mathrm{PbTe}$ and $\mathrm{PbSe}-$ have $\mathrm{NaCl}$ type structures (space group $\mathrm{Fm}-3 \mathrm{~m}$ ) and similar lattice constants $(\mathrm{PbTe}=6.460 \AA, \mathrm{PbSe}=6.217 \AA)$ and hence, almost similar XRD patterns. Rietveld refinement using the software FULLPROF $^{18}$ was done to obtain the lattice constants for all the samples. Figure 1(b) shows the refinement result for sample VA2. The variation of lattice constant with Se content for all the samples is shown in the inset. All the samples
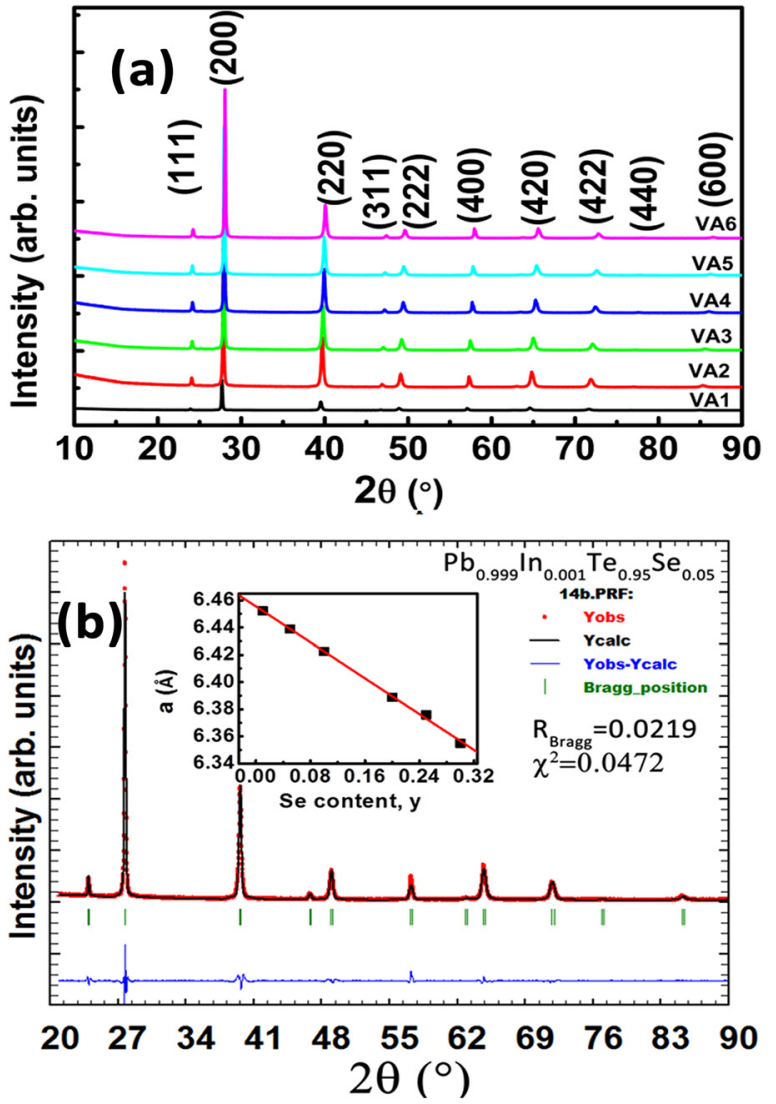

FIG. 1. (a) X-ray diffraction patterns of the samples $\mathrm{Pb}_{0.999} \mathrm{In}_{0.001} \mathrm{Te}_{1-\mathrm{y}} \mathrm{Se}_{\mathrm{y}}$ $(y=0.01,0.05,0.10,0.20,0.25$, and 0.30). (b) X-ray diffraction pattern of sample VA2 $\left(\mathrm{Pb}_{0.999} \mathrm{In}_{0.001} \mathrm{Te}_{0.95} \mathrm{Se}_{0.05}\right)$ after Rietveld refinement. The inset shows the variation of the lattice constant with Se composition for all the samples.

followed Vegard's law, which indicates solid solution formation between $\mathrm{PbTe}$ and $\mathrm{PbSe}$. The decrease in lattice constant occurs due to smaller radii of both the ions which substitute at $\mathrm{Pb}$ and $\mathrm{Te}$ sites. $\mathrm{In}^{3+}$ (ionic radius $=94 \mathrm{pm}$ ) replaces a larger $\mathrm{Pb}^{2+}$ (ionic radius $=133 \mathrm{pm}$ ) ion, while a smaller $\mathrm{Se}^{2-}$ (ionic radius $=184 \mathrm{pm}$ ) replaces the larger $\mathrm{Te}^{2-}$ (ionic radius $=207 \mathrm{pm}$ ) ion-both of which are expected to give rise to shrinkage of lattice.

Figure 2 shows scanning electron micrographs of representative sample VA2 $\left(\mathrm{Pb}_{0.999} \mathrm{In}_{0.001} \mathrm{Te}_{0.95} \mathrm{Se}_{0.05}\right)$. Figure 2(a) corresponds to the as-grown sample showing small pores in several places. Figure 2(b) shows their fracture surface, indicating the average particle size to be in micrometers. Figure 2(c) shows the polished surface of the hot press sample indicating single phase and no distinct grain boundaries. Figure 2(d) is the micrograph of fracture surface of the hot press pellet, which showed reduction of the pores and densification during the hot press. The nominal and the observed composition for all the samples taken at several points and averaged are listed in Table I. In all the samples, even though within error limits, a slight excess of $\mathrm{Pb}$ was observed. The observed amount of selenium and tellurium matched well with the nominal composition, while indium was found to be lower. The loss of indium due to high vapor pressure (v.p.) has been ruled out by comparing with v.p. of other elements at high temperatures. At $1233 \mathrm{~K}$, indium 

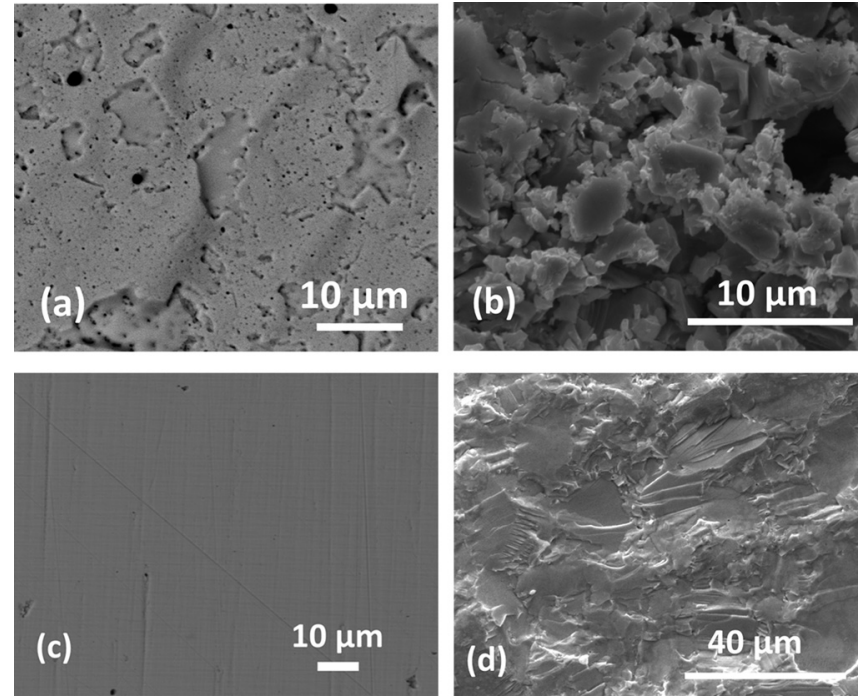

FIG. 2. Scanning electron micrographs of representative sample VA2 $\left(\mathrm{Pb}_{0.999} \mathrm{In}_{0.001} \mathrm{Te}_{0.95} \mathrm{Se}_{0.05}\right)$ showing (a) as-grown sample (b) fracture surface of as-grown sample (c) polished surface of hot pressed pellet and (d) fracture surface of hot pressed pellet.

achieved a vapor pressure of $1.35 \times 10^{-2}$ torr, ${ }^{19}$ while Te and $\mathrm{PbTe}$ measure at $\sim 0.63$ torr and $\sim 1 \times 10^{-2}$ torr, respectively, at $1215 \mathrm{~K} .{ }^{20}$ Since Te has a higher v.p. than indium and could thus vaporize more easily during synthesis, but was found to be present in almost similar values as the nominal composition, it is less probable that In would be lost in the synthesis process. Instead, the less amount of In observed here could be because it had been added in extremely small amount $(0.1$ at. \%), and it is difficult to obtain very accurate values for the same from using internal standards during EPMA measurement.

\section{B. Transport properties}

Figure 3 shows temperature dependent Seebeck coefficient from $300-800 \mathrm{~K}$. All the samples were of n-type indicating electrons as the majority carriers throughout the temperature range. The values from literature for several related $\mathrm{PbTe}$ and $\mathrm{PbSe}$ systems ${ }^{8,10,21,22}$ are plotted alongside the experimental data. The Seebeck coefficient obtained in this study resembles more closely to reports on $\mathrm{PbSe}$ system and iodine doped n-type $\mathrm{PbTe}$, which is a better n-type donor as compared to indium in PbTe. This is because of similar carrier concentration $\left(4 \times 10^{19} \mathrm{~cm}^{-3}\right.$ to $\left.5 \times 10^{19} \mathrm{~cm}^{-3}\right)$ obtained in this study. For In doped PbTe, (Gelbstein et al. ${ }^{21}$

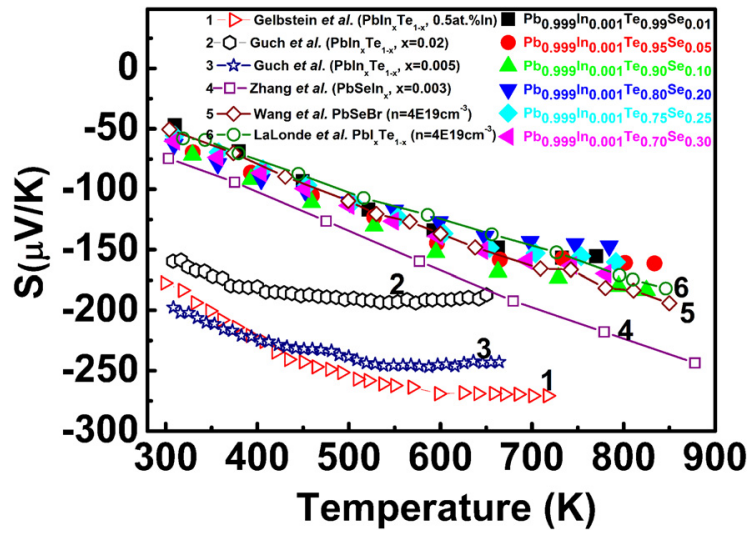

FIG. 3. Temperature dependent Seebeck coefficient for the samples $\mathrm{Pb}_{0.999} \mathrm{In}_{0.001} \mathrm{Te}_{1-\mathrm{y}} \mathrm{Se}_{\mathrm{y}}(\mathrm{y}=0.01,0.05,0.10,0.20,0.25$, and 0.30$)$. The literature values are plotted alongside (open symbols) for comparison.

and Guch et al. ${ }^{22}$ ) higher Seebeck coefficient was reported throughout the temperature range corresponding to larger room temperature values $(\sim-150$ to $-200 \mu \mathrm{V} / \mathrm{K})$ due to their lower carrier concentration $\left(\sim 10^{18} \mathrm{~cm}^{-3}\right)$ as compared to $\mathrm{n} \sim 10^{19} \mathrm{~cm}^{-3}$ reported for the halogen doped $\mathrm{PbTe}^{10}$ and PbSe. ${ }^{9}$ The Seebeck values for all the samples do not show any systematic variation with Se content since the carrier concentration does not changes systematically, as seen later in the results obtained by Hall measurement. Figure 4 shows the carrier concentration (n) from 300-800 K. The samples do not show much change in $\mathrm{n}$ on variation of Se content since the indium content was kept constant in all the samples and unlike halogens, Se belongs to the same group as Te so that no extra electrons are given to the $\mathrm{PbTe}_{1-\mathrm{y}} \mathrm{Se}_{\mathrm{y}}$ system. The observed minor differences might be due to defects, dislocations etc., occurring during synthesis which cannot be quantified easily and also due to a slight $\mathrm{Te} / \mathrm{Se}$ loss resulting in off-stoichiometry. Since the solubility of $\mathrm{Pb}$ is very less in $\mathrm{PbTe}$, even a slight excess of $\mathrm{Pb}$ might give excess of electrons, which would have resulted in the higher carrier concentration observed here than that expected from only In doping. The temperature dependence of $n$ is directly related to the temperature dependence of Seebeck coefficient. This is because $\mathrm{n}$ remains constant till $700 \mathrm{~K}$, which agrees with linear increase in Seebeck. The rise in $\mathrm{n}$ values above $700 \mathrm{~K}$ is responsible for the apparent flattening of Seebeck values.

Figure 5 shows the temperature dependent resistivity from $300-800 \mathrm{~K}$. An increase in magnitude with increasing temperature shows the highly doped nature of the samples. The resistivity values obtained here are comparable to those

TABLE I. Results from compositional analysis, fitting of $\log \mu$-log T curves and the respective carrier concentration at room temperature.

\begin{tabular}{|c|c|c|c|c|c|c|}
\hline \multirow[b]{2}{*}{ S. No. } & \multirow[b]{2}{*}{ Sample } & \multirow[b]{2}{*}{ Nominal composition } & \multirow[b]{2}{*}{ EPMA composition } & \multicolumn{2}{|c|}{ Slope from $\log \mu-\log \mathrm{T}$ fit } & \multirow[b]{2}{*}{$\mathrm{n}($ at $300 \mathrm{~K})\left(10^{19} \mathrm{~cm}^{-3}\right)$} \\
\hline & & & & Region I (low T) & Region II (high T) & \\
\hline 1. & VA1 & $\mathrm{Pb}_{0.999} \mathrm{In}_{0.001} \mathrm{Te}_{0.99} \mathrm{Se}_{0.01}$ & $\mathrm{~Pb}_{1.149} \mathrm{In}_{0.0001} \mathrm{Te}_{1.002} \mathrm{Se}_{0.007}$ & -0.96 & -2.64 & 4.353 \\
\hline 2. & VA2 & $\mathrm{Pb}_{0.999} \mathrm{In}_{0.001} \mathrm{Te}_{0.95} \mathrm{Se}_{0.05}$ & $\mathrm{~Pb}_{1.147} \mathrm{In}_{0.002} \mathrm{Te}_{0.947} \mathrm{Se}_{0.049}$ & -0.85 & -2.51 & 3.911 \\
\hline 3. & VA3 & $\mathrm{Pb} \mathrm{b}_{0.999} \mathrm{In}_{0.001} \mathrm{Te}_{0.90} \mathrm{Se}_{0.10}$ & $\mathrm{~Pb}_{1.096} \mathrm{In}_{0.0008} \mathrm{Te}_{0.896} \mathrm{Se}_{0.095}$ & -0.78 & -3.02 & 5.006 \\
\hline 4. & VA4 & $\mathrm{Pb}_{0.999} \mathrm{In}_{0.001} \mathrm{Te}_{0.80} \mathrm{Se}_{0.20}$ & $\mathrm{~Pb}_{1.129} \mathrm{In}_{0.0008} \mathrm{Te}_{0.800} \mathrm{Se}_{0.182}$ & -0.97 & -1.72 & 4.178 \\
\hline 5. & VA5 & $\mathrm{Pb}_{0.999} \mathrm{In}_{0.001} \mathrm{Te}_{0.75} \mathrm{Se}_{0.25}$ & $\mathrm{~Pb}_{1.098} \mathrm{In}_{0.0002} \mathrm{Te}_{0.737} \mathrm{Se}_{0.288}$ & -0.59 & -2.63 & 3.901 \\
\hline 6. & VA6 & $\mathrm{Pb}_{0.999} \mathrm{In}_{0.001} \mathrm{Te}_{0.70} \mathrm{Se}_{0.30}$ & $\mathrm{~Pb}_{1.0375} \mathrm{In}_{0.0005} \mathrm{Te}_{0.699} \mathrm{Se}_{0.267}$ & -1.11 & -2.46 & 4.401 \\
\hline
\end{tabular}




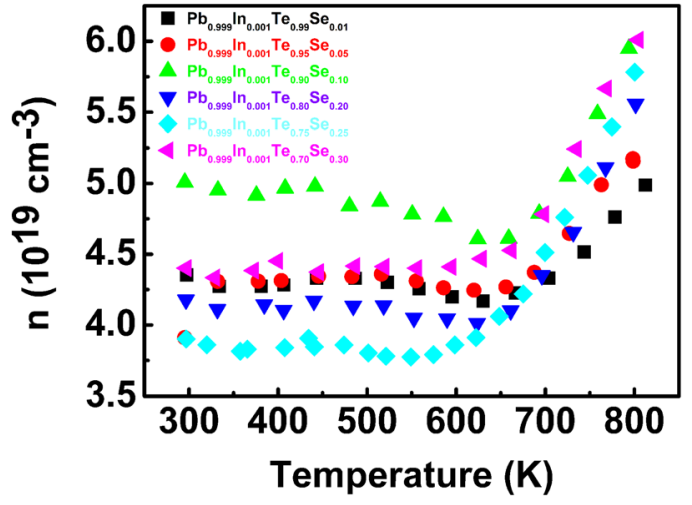

FIG. 4. Temperature dependent carrier concentration for the samples $\mathrm{Pb}_{0.999} \mathrm{In}_{0.001} \mathrm{Te}_{1-\mathrm{y}} \mathrm{Se}_{\mathrm{y}}(\mathrm{y}=0.01,0.05,0.10,0.20,0.25$, and 0.30$)$.

obtained by Wang et al. ${ }^{9}$ for a similar carrier concentration in $\mathrm{Br}$ doped $\mathrm{PbTe}-\mathrm{PbSe}$ alloys and lower than that of Gelbstein et al. ${ }^{21}$ for 0.1 at. \% In doped PbTe. To some extent, with increase of selenium content, the resistivity increases, in contrast to Seebeck results.

Figure 6 shows Pisarenko plots $\left(\mathrm{S}-\mathrm{n}_{\mathrm{H}}\right)$ at different temperatures. $\mathrm{PbTe}$ and $\mathrm{PbSe}$ are narrow band gap materials with a direct band gap at $\mathrm{L}$ point of the Brillouin zone and the importance of nonparabolicity of bands is important for their alloys too. ${ }^{23}$ Therefore, the theoretical curves here are obtained from single Kane band (SKB) model described in Refs. 9 and 24, assuming conduction by a single band for which the transport coefficients are given as follows:

The Seebeck coefficient is given by

$$
\mathrm{S}=\frac{\mathrm{k}_{\mathrm{B}}}{\mathrm{e}}\left[\frac{{ }^{1} \mathrm{~F}_{-2}^{1}}{{ }^{0} \mathrm{~F}_{-2}^{1}}-\xi\right]
$$

where e is the electronic charge, $\xi$ is the reduced chemical potential, ${ }^{1} \mathrm{~F}_{-2}^{1}$ is the integral of the form

$$
{ }^{n} \mathrm{~F}_{1}^{\mathrm{m}}=\int_{0}^{\infty}\left(-\frac{\partial \mathrm{f}}{\partial \varepsilon}\right) \varepsilon^{\mathrm{n}}\left(\varepsilon+\alpha \varepsilon^{2}\right)^{\mathrm{m}}\left[(1+2 \alpha \varepsilon)^{2}+2\right]^{1 / 2} \mathrm{~d} \varepsilon,
$$

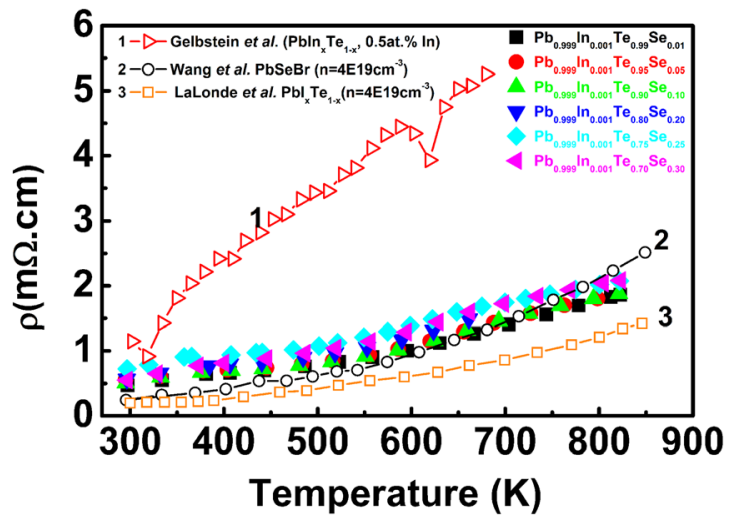

FIG. 5. Temperature dependent electrical resistivity for the samples $\mathrm{Pb}_{0.999} \mathrm{In}_{0.001} \mathrm{Te}_{1-\mathrm{y}} \mathrm{Se}_{\mathrm{y}}(\mathrm{y}=0.01,0.05,0.10,0.20,0.25$, and 0.30$)$. The literature values are plotted alongside (open symbols) for comparison.

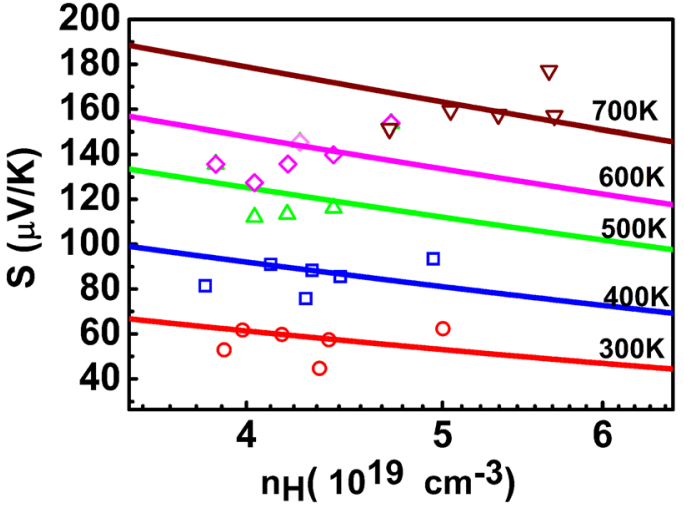

FIG. 6. The plots of $\mathrm{S}-\mathrm{n}_{\mathrm{H}}$ (Pisarenko plots) for different temperatures. The open symbols correspond to the experimental data for all the samples $\mathrm{Pb}_{0.999} \mathrm{In}_{0.001} \mathrm{Te}_{1-\mathrm{y}} \mathrm{Se}_{\mathrm{y}}(\mathrm{y}=0.01,0.05,0.10,0.20,0.25$, and 0.30$)$ at each temperature, described as follows: $\bigcirc-300 \mathrm{~K}, \square-400 \mathrm{~K}, \triangle-500 \mathrm{~K}, \diamond-$ $600 \mathrm{~K}$, and $\nabla-700 \mathrm{~K}$. The curves have been calculated theoretically.

where $\mathrm{f}$ is the Fermi function, $\varepsilon$ is the carrier energy and $\alpha=\mathrm{k}_{\mathrm{B}} \mathrm{T} / \mathrm{E}_{\mathrm{g}}$. For lead chalcogenides, the band gap $\left(\mathrm{E}_{\mathrm{g}}\right)$ is temperature dependent, given by

$$
\varepsilon_{\mathrm{g}} / \mathrm{eV}=0.17+4 \times 10^{-4} \mathrm{~T} / \mathrm{K} .
$$

Carrier density is given by

$$
\mathrm{n}=\frac{\left(2 \mathrm{~m}^{*} \mathrm{k}_{\mathrm{B}} \mathrm{T}\right)^{3 / 2}}{3 \pi^{2} \hbar^{3}}{ }^{0} \mathrm{~F}_{0}^{3 / 2}
$$

and mobility by

$$
\mu=\frac{2 \pi \hbar^{4} \mathrm{eC}_{1}}{\mathrm{~m}_{\mathrm{I}}^{*}\left(2 \mathrm{~m}_{\mathrm{b}}^{*} \mathrm{k}_{\mathrm{B}} \mathrm{T}\right)^{3 / 2} \Xi^{2}} \frac{3^{0} \mathrm{~F}_{-2}^{1}}{{ }^{0} \mathrm{~F}_{0}^{3 / 2}},
$$

where $\hbar$ is the reduced Planck's constant, $\mathrm{C}_{1}$ the average longitudinal elastic constant, $\mathrm{m}_{\mathrm{I}}^{*}=3\left(\frac{2}{\mathrm{~m}_{\perp}^{*}}+\frac{1}{\mathrm{~m}_{\|}^{*}}\right)^{-1}$ is the inertial effective mass, $\Xi$ is the deformation potential and $\mathrm{m}^{*}=\mathrm{N}_{\mathrm{V}}^{2 / 3} \mathrm{~m}_{\mathrm{b}}^{*}$. The values for all the constants have been taken from Ref. 9. The values of $\mathrm{n}$ and $\mu$ were multiplied by Hall factor (A), defined by

$$
\mathrm{A}=\frac{3 \mathrm{~K}(\mathrm{~K}+2)}{(2 \mathrm{~K}+1)^{2}} \frac{\mathrm{F}_{-4}^{1 / 2}{ }^{0} \mathrm{~F}_{0}^{3 / 2}}{\left({ }^{0} \mathrm{~F}_{-2}^{1}\right)^{2}},
$$

where the factor $\mathrm{K}$ is the ratio of effective masses $\left(\mathrm{K}=\mathrm{m}_{\|}^{*} / \mathrm{m}_{\perp}^{*}=1.75\right)$, assuming $\mathrm{T}$ independence and $\frac{3 \mathrm{~K}(\mathrm{~K}+2)}{(2 \mathrm{~K}+1)^{2}}$ is the anisotropy factor. This is because the Hall factor accounts for the nonspherical shape of the constant energy surfaces of lead chalcogenides as well as the dependence of relaxation time on energy ${ }^{24}$ such that its value deviates from 1. Figure 7 shows the dependence of A on $\eta$ at different temperatures. The increase of these values for higher $\mathrm{T}$ and high $\eta$ values shows that the usual assumption of $\mathrm{A}=1$ is not valid in this system too, so that the Hall carrier concentration $\left(n_{H}=n / A\right)$ was used for all the calculations. At lower temperatures, the Pisarenko plots describe the data well, which indicated that In does not shows any resonant 


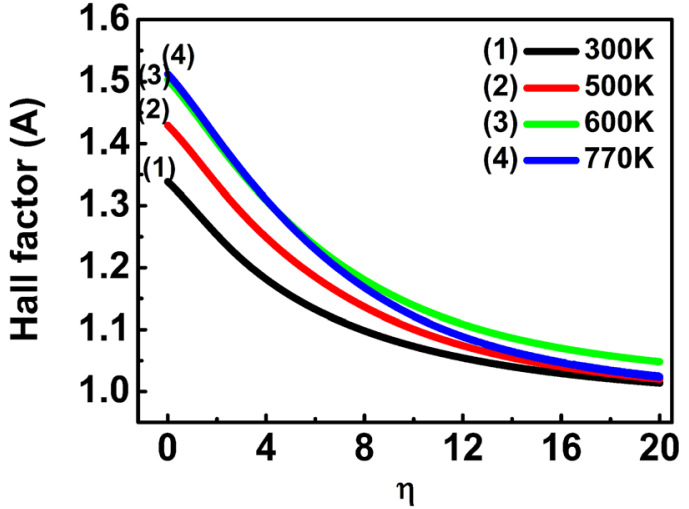

FIG. 7. Hall factor as a function of reduced Fermi energy at different temperatures.

behavior in $\mathrm{Pb}_{0.999} \mathrm{In}_{0.001} \mathrm{Te}_{1-\mathrm{y}} \mathrm{Se}_{\mathrm{y}}(\mathrm{y}=0.01,0.05,0.10,0.20$, 0.25 , and 0.30$)$ system, unlike other reports of In doping on $\mathrm{SnTe}^{25} \mathrm{PbSn}_{\mathrm{x}} \mathrm{Te}_{1-\mathrm{x}}(\mathrm{x}=0.18)$, etc. This might be because the band structure of $\mathrm{PbTe}_{1-\mathrm{y}} \mathrm{Se}_{\mathrm{y}}$ alloy is not much different from $\mathrm{PbTe}$ and $\mathrm{PbSe}$. The $\ln \left(\mathrm{m}^{*}\right)$ vs $\ln (\mathrm{T})$ curve is given in Fig. 8. The slope is 0.56 which agrees with the theoretical $\frac{\mathrm{d}(\ln m *)}{\mathrm{d}(\ln \mathrm{T})}=0.5$ dependence for lead chalcogenides. This strong temperature dependence of $\mathrm{m}^{*}$ on $\mathrm{T}$ occurs due to both interactions between the conduction and valence band, thermal expansion of the lattice and interaction between carriers and phonons. $^{24}$

Figure 9 shows the temperature dependent mobility for all the samples. All the samples showed power law decrease $\mu=\mathrm{T}^{\nu}$ with temperature, which arises because of the energy and temperature dependence of relaxation time $(\tau)$ and $\mathrm{m}^{*}$, respectively. The coefficient $\nu$ showed two different set of values (Table I) showing scattering by acoustic phonons, as expected at high temperatures. The $\nu \sim 1$ can be accounted for by a mixed scattering mechanism of acoustic phonon and ionized impurity scattering. At higher temperatures, all the lead chalcogenides show $\mathrm{T}^{-2.5}$ behavior for acoustic scattering due $\mathrm{m}^{*(-2)}$ temperature dependence in the relation $\mu \propto \mathrm{m}^{*(-2)} \mathrm{T}^{-1}$ for degenerate semiconductors. ${ }^{24}$ The mobility values at all temperatures in this study are lower than those observed in n-type $\mathrm{PbSe},{ }^{7,9,26} \mathrm{PbTe}^{10}$ first, because In

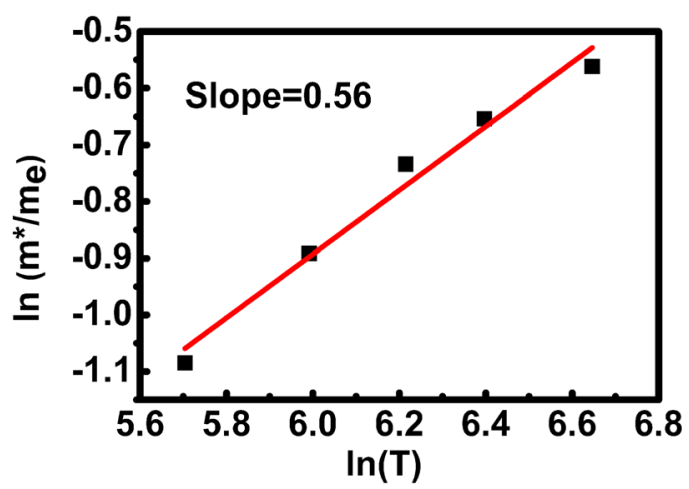

FIG. 8. Plot of $\ln \left(\mathrm{m}^{*}\right)-\ln (\mathrm{T})$. Each point in the graph corresponds to the effective mass for the $\mathrm{Pb}_{0.999} \mathrm{In}_{0.001} \mathrm{Te}_{1-\mathrm{y}} \mathrm{Se}_{\mathrm{y}}(\mathrm{y}=0.01,0.05,0.10,0.20,0.25$, and 0.30 ) series of samples calculated from the $S-n_{H}$ curves at different temperatures.

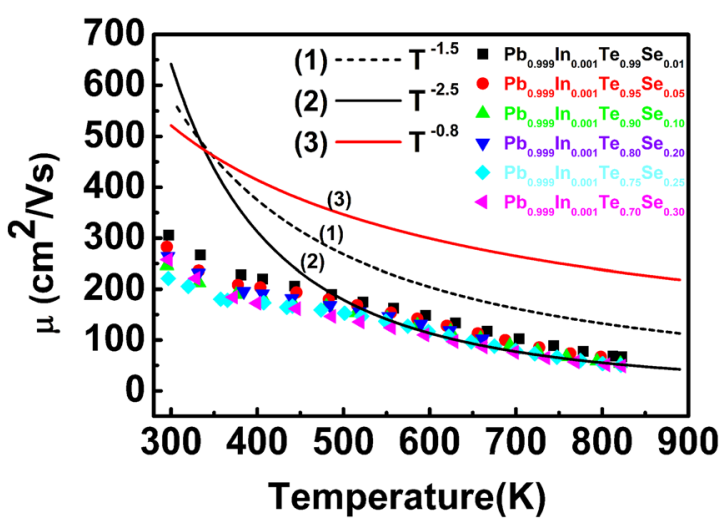

FIG. 9. Temperature dependent mobility for the samples $\mathrm{Pb}_{0.999} \mathrm{In}_{0.001} \mathrm{Te}_{1-\mathrm{y}} \mathrm{Se}_{\mathrm{y}}$ $(\mathrm{y}=0.01,0.05,0.10,0.20,0.25$, and 0.30$)$.

does not completely dope in $\mathrm{PbTe}_{1-\mathrm{y}} \mathrm{Se}_{\mathrm{y}}$ as compared to other n-type dopants like halogens $(\mathrm{Br}, \mathrm{I})$ or p-type dopants like $\mathrm{Na}, \mathrm{K}$ etc., and second, due to alloy scattering. With increase in temperature, decrease in mobility is observed in all samples.

Figure 10(a) shows the temperature dependent total thermal conductivity for all the samples. The electronic part was calculated using the Wiedemann-Franz relation $\kappa_{\mathrm{e}}=\mathrm{LT} / \rho$, where $\mathrm{L}$ is the Lorenz number. The lattice contribution (Fig. 10(b)) was then obtained by subtracting it from $\kappa_{\text {total }}$. Since the electronic contribution is affected by the nonparabolicity of the bands, Lorenz number was calculated from SKB model assuming acoustic phonon scattering using the equation, ${ }^{9,24}$
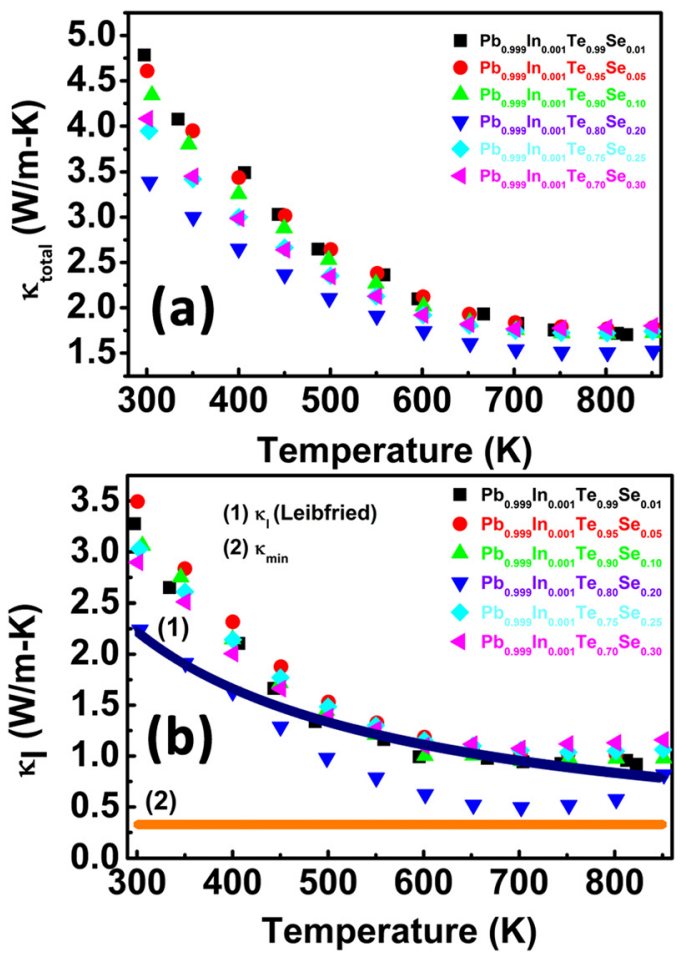

FIG. 10. (a) Temperature dependent total thermal conductivity for the samples $\mathrm{Pb}_{0.999} \mathrm{In}_{0.001} \mathrm{Te}_{1-\mathrm{y}} \mathrm{Se}_{\mathrm{y}}(\mathrm{y}=0.01,0.05,0.10,0.20,0.25$, and 0.30$)$. (b) Temperature dependent lattice thermal conductivity for the samples $\mathrm{Pb}_{0.999} \mathrm{In}_{0.001} \mathrm{Te}_{1-\mathrm{y}} \mathrm{Se}_{\mathrm{y}}(\mathrm{y}=0.01,0.05,0.10,0.20,0.25$, and 0.30$)$. The curves indicate the theoretically calculated curves. 


$$
\mathrm{L}=\left(\frac{\mathrm{k}_{\mathrm{B}}}{\mathrm{e}}\right)^{2}\left[\frac{{ }^{2} \mathrm{~F}_{-2}^{1}}{{ }^{0} \mathrm{~F}_{-2}^{1}}-\left(\frac{{ }^{1} \mathrm{~F}_{-2}^{1}}{{ }^{0} \mathrm{~F}_{-2}^{1}}\right)^{2}\right],
$$

where the parameters have already been described. At $300 \mathrm{~K}$, $\kappa_{1}$ values varied between 4.7 and $3.4 \mathrm{~W} / \mathrm{mK}$, which is slightly higher as compared to other reports. ${ }^{9-11}$ At high temperatures, $\kappa_{1}$ values reduce to $\sim 1 \mathrm{~W} / \mathrm{m}-\mathrm{K}$, which is still high as compared to the theoretically calculated minimum $\kappa_{1}$ of $0.38 \mathrm{~W} / \mathrm{m}-\mathrm{K}$ possible for PbTe at its melting point, ${ }^{27}$ considering both the longitudinal and optical branches. This shows that there is a further scope in such compounds to reduce the lattice thermal conductivity, possibly by reducing grain size, nanostructuring, etc., to achieve higher $z$ T. Microstructural engineering approach has been previously used ${ }^{28-33}$ for several thermoelectric materials. The expected decrease in $\kappa_{1}$ with temperature due to Umklapp scattering was observed upto $\sim 700 \mathrm{~K}$. The possible role of indium doping here could be to scatter phonons to reduce thermal conductivity.

A simple model by Leibfried and Schlömann for $\kappa_{1}$ dependence at high temperature is given by ${ }^{34}$

$$
\kappa_{1}=3.5\left(\frac{\mathrm{k}_{\mathrm{B}}}{\mathrm{h}}\right)^{3} \frac{\mathrm{MV}^{1 / 3} \Theta_{\mathrm{D}}^{3}}{\gamma^{2} \mathrm{~T}},
$$

where $\mathrm{M}$ is the average mass per atom, $\mathrm{V}$ the average volume per atom, $\gamma$ the Gruneisen parameter, which shows the anharmonic nature of the crystal and $\Theta_{D}$ is the Debye temperature. Here, the volume was taken from the Rietveld refinement for sample VA2, while $\Theta_{\mathrm{D}}=136 \mathrm{~K}$ (Ref. 35) and $\gamma=1.45$ for $\mathrm{PbTe}$ since the content of $\mathrm{Te}$ is higher at this composition. Since this model does not explicitly consider different scattering mechanisms, it shows only $1 / \mathrm{T}$ decrease, but may not agree accurately if any specific scattering mechanism dominates. Second, in $\mathrm{PbTe}_{1-\mathrm{y}} \mathrm{Se}_{\mathrm{y}}$ solid solutions, with increase of Se content, $\kappa_{1}$ first decreases, comes to a minimum at $\sim y=0.5$, and then rises again ${ }^{26,36}$ due to highest disorder at mid compositions. Here, electron-phonon interaction can be neglected due to low values of $\kappa_{\mathrm{e}}$ while scattering by acoustic and also by optical polar phonons are considered.

Figure 11 shows the thermoelectric figure of merit $(z \mathrm{~T})$ for all the samples. The highest $z \mathrm{~T}$ of 0.66 was achieved at $800 \mathrm{~K}$ for sample with $30 \%$ Se content due to optimization

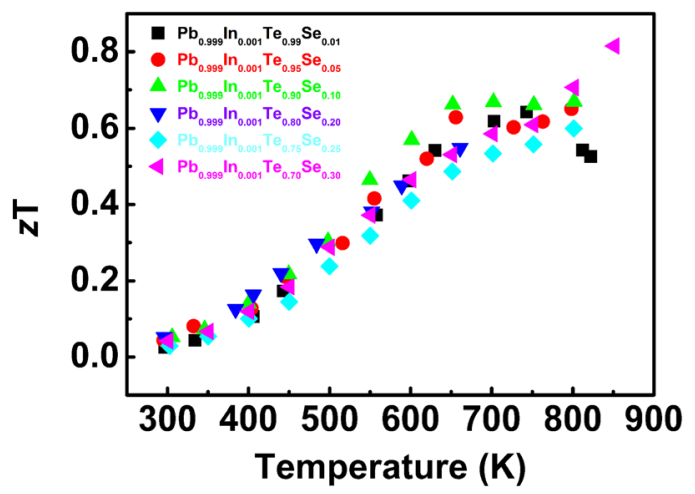

FIG. 11. Temperature dependent thermoelectric figure of merit $(z \mathrm{~T})$ for the samples $\mathrm{Pb}_{0.999} \mathrm{In}_{0.001} \mathrm{Te}_{1-\mathrm{y}} \mathrm{Se}_{\mathrm{y}}(\mathrm{y}=0.01,0.05,0.10,0.20,0.25$, and 0.30). of the carrier concentration for these samples. This is higher than 0.45 at $650 \mathrm{~K}$ obtained by Guch et al. for In doped PbTe but lower than 0.92 at $575 \mathrm{~K}$ by Gelbstein and $\sim 1.0$ at $850 \mathrm{~K}$ by Wang et al. for $\mathrm{Br}$ doped $\mathrm{PbSe}$. A possible reason is the occurrence of bipolar effect at high temperatures in the present set of samples, which was absent in all the other reports.

\section{CONCLUSIONS}

A number of n-type indium doped $\mathrm{PbTe}_{1-\mathrm{y}} \mathrm{Se}_{\mathrm{y}}$ alloys were synthesized by solid state synthesis method and the thermoelectric properties were studied. Indium was not found to lead to Fermi level pinning in $\mathrm{PbTe}_{1-\mathrm{y}} \mathrm{Se}_{\mathrm{y}}$ as previously reported in $\mathrm{PbTe}$. Indium is also not a resonant scatterer in these alloys. Bipolar conduction was found at high temperature which was not observed in $\mathrm{PbTe}$ or $\mathrm{PbSe}$ doped with halogens (good dopant) for n-type or $\mathrm{Na}$ doping for p-type materials. This indicates that it is very important to control the carrier concentration in the alloys for good thermoelectric properties, particularly at high temperatures. Future work could involve proper control of carrier concentration to avoid bipolar contribution at high temperature, and a further reduction of lattice thermal conductivity by other approaches like nanostructuring, which could increase the $z \mathrm{~T}$ further.

\section{ACKNOWLEDGMENTS}

One of the authors (AB) would like to acknowledge IndoUS Science and Technology Forum (IUSSTF) and American Physical Society (APS) for the grant received under the "APS-IUSSTF Physics Student Visitation Program."

${ }^{1}$ Z. H. Dughaish, Physica B 322, 205 (2002).

${ }^{2}$ V. Jovovic, S. J. Thiagarajan, J. P. Heremans,T. Komissarova, D. Khokhlov, and A. Nicorici, J. Appl. Phys 103, 053710 (2008).

${ }^{3}$ Y. Pei, X. Shi, A. LaLonde, H. Wang, L. Chen, and G. J. Snyder, Nature 473, 66 (2011).

${ }^{4}$ R. N. Tauber and I. B. Cadoff, J. Appl. Phys 38, 3714 (1967).

${ }^{5}$ J. Steinnger, Metall. Trans. 1, 2939 (1970).

${ }^{6}$ I. Kudman, J. Mater. Sci. 7, 1027 (1972).

${ }^{7}$ H. Wang, Y. Pei, A. D. LaLonde, and G. J. Snyder, Adv. Mater. 23, 1366 (2011).

${ }^{8}$ Q. Zhang, F. Cao, W. Liu, K. Lukas, B. Yu, S. Chen, C. Opeil, D. Broido, G. Chen, and Z. Ren, J. Am. Chem. Soc. 134, 10031 (2012).

${ }^{9}$ H. Wang, Y. Pei, A. D. LaLonde, and G. J. Snyder, Proc. Natl. Acad. Sci. USA 109, 9705 (2012).

${ }^{10}$ A. D. LaLonde, Y. Pei, and G. J. Snyder, Energy Environ. Sci. 4, 2090 (2011).

${ }^{11}$ Y. Pei, A. D. LaLonde, N. A. Heinz, and G. J. Snyder, Adv. Energy Mater. 2, 670 (2012)

${ }^{12}$ B. A. Volkov, L. I. Ryabova, and D. R. Khokhlov, Phys. Usp. 45, 819 (2002).

${ }^{13}$ D. Khokhlov and B. Volkov, HAIT J. Sci. Eng. 1, 266 (2003).

${ }^{14}$ J. P. Heremans, V. Jovovic, E. S. Toberer, Ali Saramat, K. Kurosaki, A. Charoenphakdee, S. Yamanaka, and G. J. Snyder, Science 321, 554 (2008).

${ }^{15}$ M. A. Aliyanov, Kh. I. Abilov, and B. B. Kuligv, Phys. Status Solidi A 20, K17 (1973).

${ }^{16}$ S. Iwanaga, E. S. Toberer, A. LaLonde, and G. J. Snyder, Rev. Sci. Instrum. 82, 063905 (2011).

${ }^{17}$ Y. Pei, A. D. LaLonde, H. Wang, and G. J. Snyder, Energy Environ. Sci. 5, 7963 (2012).

${ }^{18}$ J. A. Rodriguez-Carvajal, in Satellite Meeting on Powder Diffraction of the XV IUCr Congress; Vol. 127 (Toulouse, France, 1990).

${ }^{19}$ J. S. Anderson, J. Chem. Soc. 141-143 (1943).

${ }^{20}$ R. F. Bis, J. Phys. Chem. Solids 24, 579 (1963). 
${ }^{21}$ Y. Gelbstein, Z. Dashevsky, and M. P. Dariel, Physicsa B 363, 196-205 (2005).

${ }^{22}$ M. Guch, C. R. Sankar, J. Salvador, G. Meisner, and H. Kleinke, Sci. Adv. Mater. 3, 615 (2011).

${ }^{23}$ R. I. Ravich, B. A. Efimova, and V. I. Tamarchenko, Phys. Status Solidi B 43, 11 (1971)

${ }^{24}$ Y. I. Ravich, B. A. Efimova, and I. A. Smirnov, Semiconducting Lead Chalcogenides (Plenum Press, New York, 1970).

${ }^{25}$ Q. Zhang, B. Liao, Y. Lan, K. Lukas, W. Liu, K. Esfarjani, C. Opeil, D. Broido, G. Chen, and Z. Ren, Proc. Natl. Acad. Sci. 110, 13261 (2013).

${ }^{26}$ J. Androulakis, D.-Y. Chung, X. Su, L. Zhang, C. Uher, T. C. Hassapis, E. Hatzikraniotis, K. M. Paraskevopoulos, and M. G. Kanatzidis, Phys. Rev. B 84, 155207 (2011).

${ }^{27}$ D. M. Rowe and C. M. Bhandari, Modern Thermoelectrics (Reston Publishing, 1983)

${ }^{28}$ W. Kim, J. Zide, A. Gossard, D. Klenov, S. Stemmer, A. Shakouri, and A. Majumdar, Phys. Rev. Lett. 96, 045901 (2006).
${ }^{29}$ J. Androulakis, K. F. Hsu, R. Pcionek, H. Kong, C. Uher, J. J. D'Angelo, A. Downey, T. Hogan, and M. G. Kanatzidis, Adv. Mater. 18, 1170 (2006).

${ }^{30}$ P. F. P. Poudeu, J. D. Angelo, A. D. Downey, J. L. Short, T. P. Hogan, and M. G. Kanatzidis, Angew. Chem. Int. Ed. 45, 3835 (2006).

${ }^{31}$ A. J. Minnich, M. S. Dresselhaus, Z. F. Ren, and G. Chen, Energy Environ. Sci. 2, 466 (2009).

${ }^{32}$ C. J. Vineis, A. Shakouri, A. Majumdar, and M. G. Kanatzidis, Adv. Mater. 22, 3970 (2010).

${ }^{33}$ J.-F. Li, W.-S. Liu, L.-D. Zhao, and M. Zhou, NPG Asia Mater. 2, 152 (2010).

${ }^{34}$ H. J. Goldsmid, Electronic Refrigeration (Pion, 1986), p. 73.

${ }^{35}$ O. Madelung, Semiconductors: Data Handbook (Springer-Verlag, Berlin, 2003).

${ }^{36}$ H. Wang, A. D. LaLonde, Y. Pei, and G. J. Snyder, Adv. Funct. Mater. 23, 1586 (2013). 